\title{
POISSON-LIE STRUCTURE ON THE TANGENT BUNDLE OF A POISSON-LIE GROUP, AND POISSON ACTION LIFTING
}

\author{
MOHAMED BOUMAIZA AND NADHEM ZAALANI
}

Communicated by Charles-Michel Marle

Abstract. We show in this paper that the tangent bundle $T G$, of a Poisson-Lie group $G$ has a Poisson-Lie group structure given by the canonical lifting of that of $G$. We determine the dual group of $T G$, its Lie bialgebra and its double Lie algebra.

We also show that any Poisson action of $G$ on a Poisson manifold $P$ is lifted on a Poisson action of $T G$ on the tanget bundle $T P$.

\section{Introduction}

Poisson-Lie group theory was first introduced by Drinfel'd [1] [2] and SemenovTian-Shansky [11]. Semenov and Kosmann-Schwarzbach [4] used Poisson-Lie groups to understand the Hamiltonian structure of the group of dressing transformations of certain integrable systems. These Poisson-Lie groups play the role of symmetry groups. Theory of Poisson-Lie groups was remarkably developed by Weinstein [9] [13], Drinfel'd [3] and Jiang-Hua Lu [6] [7].

Let $(G, \omega)$ be a Poisson-Lie group with Lie algebra $\mathcal{G}$ and multiplication $m: G \times G \longrightarrow G$.

We assume that the tangent bundle $T G$ is equipped with the Poisson structure $\Omega_{T G}$ introduced by Sanchez de Alvarez in [10]. In this case, $T G$ has a PoissonLie group structure with dual Poisson-Lie group $\left(T G^{*}, \Omega_{T G^{*}}\right)$ and Lie bialgebra $\left(\mathcal{G} \dashv \mathcal{G}, \mathcal{G}^{*} \vdash \mathcal{G}^{*}\right)$, where $G^{*}$ is the dual of $G, \mathcal{G} \dashv \mathcal{G}$ is the semi-direct product Lie algebra with bracket

$$
\left[(x, y),\left(x^{\prime}, y^{\prime}\right)\right]=\left(\left[x, x^{\prime}\right],\left[x, y^{\prime}\right]+\left[y, x^{\prime}\right]\right), \text { where }(x, y),\left(x^{\prime}, y^{\prime}\right) \in \mathcal{G} \times \mathcal{G}
$$

and $\mathcal{G}^{*} \vdash \mathcal{G}^{*}$ is the semi-direct product Lie algebra with bracket

$\left[(\alpha, \beta),\left(\alpha^{\prime}, \beta^{\prime}\right)\right]=\left(\left[\alpha, \beta^{\prime}\right]+\left[\beta, \alpha^{\prime}\right],\left[\beta, \beta^{\prime}\right]\right)$, where $(\alpha, \beta),\left(\alpha^{\prime}, \beta^{\prime}\right) \in \mathcal{G}^{*} \times \mathcal{G}^{*}$. 
The double Lie algebra $\check{\mathcal{D}}=(\mathcal{G} \dashv \mathcal{G}) \oplus\left(\mathcal{G}^{*} \vdash \mathcal{G}^{*}\right)$, of the Poisson-Lie group $\left(T G, \Omega_{T G}\right)$ is isomorphic to the semi-direct product Lie algebra $\mathcal{D} \dashv \mathcal{D}$, where $\mathcal{D}=\mathcal{G} \oplus \mathcal{G}^{*}$ is the double Lie algebra of $(G, \omega)$.

Let $H$ be a Poisson-Lie subgroup of $(G, \omega)$. Then the tangent bundle $T H$ is also a Poisson-Lie subgroup of $\left(T G, \Omega_{T G}\right)$.

Let $P$ be a Poisson manifold and $\phi: G \times P \longrightarrow P$, be a Poisson action of $G$ on $P$. Then $\phi$ has a lifted Poisson action of the Poisson Lie group $T G$ on the Poisson manifold TP. As example of Poisson action we consider the dressing action [7]. In this case we show that the lifted action of the left dressing action of $G^{*}$ on $G$ is also the left dressing action of $T G^{*}$ on $T G$.

\section{Poisson-Lie Structure on the Tangent Bundle of a Poisson-Lie Group}

The notion of a Poisson-Lie group is due to Drinfiel'd [1]. Let us recall its definition and some properties.

Definition 1. A Poisson-Lie group is a Lie group G, equipped with a Poisson structure $\omega$ such that the product

$$
m: G \times G \longrightarrow G:(g, h) \longmapsto m(g, h)=g h
$$

is a Poisson map, where $G \times G$ is equipped with the product Poisson structure.

The Poisson tensor $\omega$ of a Poisson-Lie group $G$ vanishes at the unit element $e$ of $G$. Its derivative, $\mathrm{d}_{e} \omega \in \mathcal{G} \wedge \mathcal{G}$, at that point is a 1-cocycle of $\mathcal{G}$ relative to the adjoint representation of $\mathcal{G}$ on $\mathcal{G} \wedge \mathcal{G}$. Then, there exists a Lie bracket on $\mathcal{G}^{*}$ given by

$$
\left\langle[\alpha, \beta]_{\omega}, x\right\rangle=\mathrm{d}_{e} \omega(x)(\alpha, \beta)
$$

where $x \in T_{e} G=\mathcal{G}, \alpha$ and $\beta \in T_{e}^{*} G=\mathcal{G}^{*}$.

The connected and simply connected Lie group $G^{*}$ with Lie algebra $\mathcal{G}^{*}$ is called the dual group of the Poisson-Lie group $G$. It has, too, a structure of Poisson-Lie group.

This dual group $G^{*}$ acted on $G$ by the (dressing action), whose orbits determine the symplectic leaves of $G$.

In this section, we give for every Poisson-Lie group $G$ a structure of Poisson Lie group on the tangent bundle $T G$. The Poisson structure on $T G$ is that given by Sanchez de Alvarez [10]. Let us recall it in the case of a Poisson manifold $P$. 
Theorem 2. [10]. Let $P$ be a Poisson manifold with Poisson bracket $\{,\}_{P}$. We denote by $\tau$ the canonical projection from $T P$ on $P$. For all $\varphi \in C^{\infty}(P)$, we denote $\hat{\varphi}=\varphi \circ \tau$ and $\dot{\varphi}$ the tangent map of $\varphi$.

Then TP has a unique Poisson structure, denoted by $\{,\}_{\text {TP }}$ such that:

i) $\{\hat{\varphi}, \hat{\psi}\}_{T P}=0$

ii) $\{\hat{\varphi}, \dot{\psi}\}_{T P}=\{\dot{\varphi}, \hat{\psi}\}_{T P}=\left\{\varphi, \psi \hat{\}}_{P}\right.$

iii) $\{\dot{\varphi}, \dot{\psi}\}_{T P}=\{\varphi, \psi\}_{P}, \quad$ for all $\varphi, \psi \in C^{\infty}(P)$.

Remark 3. [10]. Let $\left(x_{i}\right), i=1, \ldots, n$ are local coordinates of $P$, such that the bracket of $P$ is given by $\left\{x_{i}, x_{j}\right\}=\omega_{i j}(x)$. In the local coordinates $\left(x_{i}, \dot{x}_{i}\right)$ of $T P$, the bracket $\{,\}_{T P}$ is given by:

i) $\left\{x_{i}, x_{j}\right\}_{T P}=0$

ii) $\left\{\dot{x}_{i}, x_{j}\right\}_{T P}=\left\{x_{i}, \dot{x}_{j}\right\}_{T P}=\left\{x_{i}, x_{j}\right\}=\omega_{i j}(x)$

iii) $\left\{\dot{x}_{i}, \dot{x}_{j}\right\}_{T P}=\left\{x_{i}, x_{j} \dot{\}}_{P}=\dot{\omega}_{i j}(\dot{x})=\sum_{k} \frac{\partial \omega_{i j}}{\partial x_{k}}(x) \dot{x}_{k}\right.$.

Proposition 4. Let $G$ be a Lie group with Lie algebra $\mathcal{G}$. We assume that TG is equipped with the map

$$
\check{m}: T G \times T G \longrightarrow T G:\left(X_{g}, Y_{h}\right) \longmapsto L_{g *} Y_{h}+R_{h *} X_{g}
$$

Then TG is a Lie group with Lie algebra the semi-direct product of Lie algebras $\mathcal{G} \dashv \mathcal{G}$, where the bracket is given by

$$
\left[(x, y),\left(x^{\prime}, y^{\prime}\right)\right]=\left(\left[x, x^{\prime}\right],\left[x, y^{\prime}\right]+\left[y, x^{\prime}\right]\right) .
$$

It is clear that $T G$ is isomorphic to the semi-direct product Lie groups $G \dashv \mathcal{G}$, associated to the adjoint action of $G$ on the abelian Lie group $\mathcal{G}$. Then the Lie algebra of $T G$ is the semi-direct product Lie algebra $\mathcal{G} \dashv \mathcal{G}$.

With this preparation, we can give the main result of this section.

Theorem 5. Let $(G, \omega)$ be a Poisson-Lie group. We assume that TG is equipped with the multiplication

$$
X_{g} . Y_{h}=L_{g *} Y_{h}+R_{h *} X_{g}
$$

and with the Poisson structure $\{,\}_{T G}$. Then $\left(T G,\{,\}_{T G}\right)$ is a Poisson-Lie group. 
Proof: According to Definition 1, we have to show that

$$
\left\{F_{1}, F_{2}\right\}\left(X_{g} . Y_{h}\right)=\left\{F_{1 X_{g}}, F_{2 X_{g}}\right\}\left(Y_{h}\right)+\left\{F_{1 Y_{h}}, F_{2 Y_{h}}\right\}\left(X_{g}\right)
$$

for all $F_{1}, F_{2} \in C^{\infty}(T G), X_{g} \in T_{g} G$ and $Y_{h} \in T_{h} G$.

By Theorem 2, it is sufficient to consider the functions of type $\dot{\varphi}$ and $\hat{\varphi}$, where $\varphi \in C^{\infty}(P)$.

Let $\varphi, \psi \in C^{\infty}(P)$. We have

$$
\{\hat{\varphi}, \hat{\psi}\}\left(X_{g} \cdot Y_{h}\right)=\left\{\hat{\varphi}_{X_{g}}, \hat{\psi}_{X_{g}}\right\}\left(Y_{h}\right)+\left\{\hat{\varphi}_{Y_{h}}, \hat{\psi}_{Y_{h}}\right\}\left(X_{g}\right)=0 .
$$

By a simple calculation, we get

$$
\begin{aligned}
\left\{\hat{\varphi}_{X_{g}}, \dot{\psi}_{X_{g}}\right\}\left(Y_{h}\right)+\left\{\hat{\varphi}_{Y_{h}}, \dot{\psi}_{Y_{h}}\right\}\left(X_{g}\right)= & \left\{\left(\varphi \circ L_{g} \hat{)},\left(\psi \circ L_{g} \dot{j}+\hat{\alpha}\right\}\left(Y_{h}\right)\right.\right. \\
& +\left\{\left(\varphi \circ R_{h}\right),\left(\psi \circ R_{h}\right)+\hat{\beta}\right\}\left(X_{g}\right) \\
= & \{\hat{\varphi}, \dot{\psi}\}\left(X_{g} . Y_{h}\right)
\end{aligned}
$$

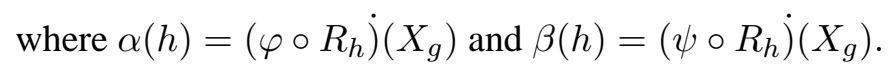

For the last bracket we have

$$
\begin{aligned}
\{\dot{\varphi}, \dot{\psi}\}\left(X_{g} \cdot Y_{h}\right) & =\{\varphi, \psi\}\left(L_{g *} Y_{h}+R_{h *} X_{g}\right) \\
& =\left(\{\varphi, \psi\} \circ L_{g}\right)\left(Y_{h}\right)+\left(\{\varphi, \psi\} \circ R_{h} \dot{)}\left(X_{g}\right) .\right.
\end{aligned}
$$

If we take $X_{g}=\sigma_{x}(g)=R_{g *} x$ and $Y_{h}=\sigma_{y}(h)$, where $x, y \in \mathcal{G}$ and $\sigma_{x}$ is the fundamental vector field associated to the left translation of $G$, we get

$$
\begin{aligned}
\left(\{\varphi, \psi\} \circ L_{g}\right)\left(\sigma_{y}(h)\right)= & \left\{\varphi \circ L_{g}, \psi \circ L_{g}\right\}\left(\sigma_{y}(h)\right) \\
& +\frac{\mathrm{d}}{\mathrm{d} t}\left\{\varphi \circ R_{\exp t y . h}, \psi \circ R_{\exp t y . h}\right\}(g)_{t=0} \\
= & \left\{\varphi \circ L_{g}, \psi \circ L_{g}\right\}\left(\sigma_{y}(h)\right)+\left\{y^{l}\left(\varphi \circ R_{h}\right), \psi \circ R_{h}\right\}(g) \\
& +\left\{\varphi \circ R_{h}, y^{l}\left(\psi \circ R_{h}\right)\right\}(g)
\end{aligned}
$$

where $y^{l}$ is the left invariant vector field whose value at $e$ is $y$.

On the other hand, we have

$$
\begin{aligned}
\left(\{\varphi, \psi\} \circ R_{h} \dot{)}\left(\sigma_{x}(g)\right)=\right. & \left\{\varphi \circ R_{h}, \psi \circ R_{h}\right\}\left(\sigma_{x}(g)\right) \\
& +\frac{\mathrm{d}}{\mathrm{d} t}\left\{\varphi \circ L_{\exp t x . g}, \psi \circ L_{\exp t x . g}\right\}_{t=o}(h) \\
= & \left\{\varphi \circ R_{h}, \psi \circ R_{h}\right\}\left(\sigma_{x}(g)\right)+\left\{\dot{\varphi}\left(\sigma_{x} \circ L_{g}\right), \psi \circ L_{g}\right\}(h) \\
& +\left\{\varphi \circ L_{g}, \dot{\psi}\left(\sigma_{x} \circ L_{g}\right)\right\}(h) .
\end{aligned}
$$


Furthermore, it is easy to verify that

$$
\begin{aligned}
& \dot{\varphi}_{\sigma_{x}(g)}\left(\sigma_{y}(h)\right)=\left(\varphi \circ L_{g}\right)^{\cdot}\left(\sigma_{y}(h)+\hat{\alpha}\left(\sigma_{y}(h)\right)\right. \\
& \dot{\varphi}_{\sigma_{y}(h)}\left(\sigma_{x}(g)\right)=\left(\varphi \circ R_{h}\right) \dot{(}\left(\sigma_{x}(g)\right)+\hat{\alpha}^{\prime}\left(\sigma_{x}(g)\right)
\end{aligned}
$$

where $\alpha^{\prime}(g)=\left(\varphi \circ L_{g}\right)\left(\sigma_{y}(h)\right)$.

Then

$$
\begin{aligned}
& \left\{\dot{\varphi}_{\sigma_{x}(g)}, \dot{\psi}_{\sigma_{x}(g)}\right\}\left(\sigma_{y}(h)\right)+\left\{\dot{\varphi}_{\sigma_{y}(h)}, \dot{\psi}_{\sigma_{y}(h)}\right\}\left(\sigma_{x}(g)\right) \\
= & \left\{\left(\varphi \circ L_{g}\right)^{\cdot}+\hat{\alpha},\left(\psi \circ L_{g}\right)^{\cdot}+\hat{\beta}\right\}\left(\sigma_{y}(h)\right)+\left\{\left(\varphi \circ R_{h}\right)^{\cdot}+\hat{\alpha}^{\prime},\left(\psi \circ R_{h}\right)^{\cdot}+\hat{\beta}^{\prime}\right\}\left(\sigma_{x}(g)\right) \\
= & \left\{\varphi \circ L_{g}, \psi \circ L_{g}\right\} \cdot\left(\sigma_{y}(h)\right)+\left\{\left(\varphi \circ L_{g}\right)^{\cdot}, \hat{\beta}\right\}\left(\sigma_{y}(h)\right)+\left\{\hat{\alpha},\left(\psi \circ L_{g}\right)^{\cdot}\right\}\left(\sigma_{y}(h)\right) \\
& +\left\{\varphi \circ R_{h}, \psi \circ R_{h}\right\}\left(\sigma_{x}(g)\right)+\left\{\hat{\alpha}^{\prime},\left(\psi \circ R_{h}\right)^{\cdot}\right\}\left(\sigma_{x}(g)\right)+\left\{\left(\varphi \circ R_{h}\right)^{\cdot}, \hat{\beta}^{\prime}\right\}\left(\sigma_{x}(g)\right) \\
= & \left\{\varphi \circ L_{g}, \psi \circ L_{g}\right\}\left(\sigma_{y}(h)\right)+\left\{\varphi \circ L_{g}, \beta\right\}(h)+\left\{\alpha, \psi \circ L_{g}\right\}(h) \\
& +\left\{\varphi \circ R_{h}, \psi \circ R_{h}\right\}\left(\sigma_{x}(g)\right)+\left\{\alpha^{\prime}, \psi \circ R_{h}\right\}(g)+\left\{\varphi \circ R_{h}, \beta^{\prime}\right\}(g) .
\end{aligned}
$$

It suffices to verify the following expressions

$$
\begin{aligned}
\alpha(h) & =\varphi^{\cdot}\left(\sigma_{x} \circ L_{g}\right)(h) \\
\beta(h) & =\dot{\psi}\left(\sigma_{x} \circ L_{g}\right)(h) \\
\alpha^{\prime}(g) & =y^{l}\left(\varphi \circ R_{h}\right)(g) \\
\beta^{\prime}(g) & =y^{l}\left(\psi \circ R_{h}\right)(g) .
\end{aligned}
$$

We replace $\alpha, \alpha^{\prime}, \beta$ and $\beta^{\prime}$ by these expressions we get

$$
\{\dot{\varphi}, \dot{\psi}\}\left(\sigma_{x}(g) . \sigma_{y}(h)\right)=\left\{\dot{\varphi}_{\sigma_{x}(g)}, \dot{\psi}_{\sigma_{x}(g)}\right\}\left(\sigma_{y}(h)\right)+\left\{\dot{\varphi}_{\sigma_{y}(h)}, \dot{\psi}_{\sigma_{y}(h)}\right\}\left(\sigma_{x}(g)\right) .
$$

This concludes the proof.

Example 6. Let $\mathcal{G}$ be a Lie algebra. We assume that $\mathcal{G}^{*}$ is equipped with its linear Poisson-Lie structure given, for all $\varphi, \psi \in C^{\infty}\left(\mathcal{G}^{*}\right)$, by

$$
\{\varphi, \psi\}(x)=\langle x,[\mathrm{~d} \varphi(x), \mathrm{d} \psi(x)]\rangle .
$$

In local coordinates $\left(x_{i}\right)$ of $\mathcal{G}^{*}$, this structure is expressed by

$$
\left\{x_{i}, x_{j}\right\}=\sum_{k} c_{i j}^{k} x_{k}
$$

where $c_{i j}^{k}$ are the structure constants of $\mathcal{G}$. 
The linear Poisson structure of $\mathcal{G}^{*} \times \mathcal{G}^{*}$ associated to the semi-direct product $\mathcal{G} \dashv \mathcal{G}$ is given by

$$
\{F, G\}(x, y)=\left\langle x,\left[\frac{\mathrm{d} F}{\mathrm{~d} x}, \frac{\mathrm{d} G}{\mathrm{~d} x}\right]\right\rangle+\left\langle y,\left[\frac{\mathrm{d} F}{\mathrm{~d} x}, \frac{\mathrm{d} G}{\mathrm{~d} y}\right]\right\rangle+\left\langle y,\left[\frac{\mathrm{d} F}{\mathrm{~d} y}, \frac{\mathrm{d} G}{\mathrm{~d} x}\right]\right\rangle
$$

for all $F, G \in C^{\infty}\left(\mathcal{G}^{*} \times \mathcal{G}^{*}\right), x, y \in \mathcal{G}^{*}$.

The local coordinates $\left(x_{i}\right)$ induce local coordinates $\left(x_{i}, y_{j}\right)$ on $\mathcal{G}^{*} \times \mathcal{G}^{*}$, such that

$$
\begin{aligned}
& \left\{y_{i}, y_{j}\right\}(x, y)=0 \\
& \left\{y_{i}, x_{j}\right\}(x, y)=\sum_{k} C_{i j}^{k} y_{k}=\omega_{i j}(y) \\
& \left\{x_{i}, x_{j}\right\}(x, y)=\sum_{k} C_{i j}^{k} x_{k}=\dot{\omega}_{i j}(x) .
\end{aligned}
$$

According to Remark 3, for the local coordinates $\left(\dot{x}_{i}, x_{j}\right)$ of $T \mathcal{G}^{*}$, this bracket coincides with that of $T \mathcal{G}^{*}$.

Hence, the Poisson-Lie group $T \mathcal{G}^{*}$ is isomorphic to the Abelien Poisson-Lie group $(\mathcal{G} \dashv \mathcal{G})^{*}$ associated to the semi-direct product Lie algebra $\mathcal{G} \dashv \mathcal{G}$.

\section{Bialgebra and Dual of the Poisson-Lie Group $T G$}

In this section, we study the infinitesimal version of the Poisson-Lie group $T G$, namely that of Lie bialgebra and double Lie algebra of $T G$.

Definition 7. [12] Let $\mathcal{G}$ be a Lie algebra with dual space $\mathcal{G}^{*}$. We say that $\left(\mathcal{G}, \mathcal{G}^{*}\right)$ form a Lie bialgebra if there is given a Lie bracket on $\mathcal{G}^{*}$ such that

$$
\langle[\alpha, \beta],[x, y]\rangle=-\left[\operatorname{ad}_{x}^{*} \alpha, \beta\right](y)-\left[\alpha, \operatorname{ad}_{x}^{*} \beta\right](y)+\left[\operatorname{ad}_{y}^{*} \alpha, \beta\right](x)+\left[\alpha, \operatorname{ad}_{y}^{*} \beta\right](x) .
$$

By Drinfel'd [1], if $(G, \omega)$ is a Poisson-Lie group, then the derivative of $\omega$ at $e$ defines a Lie algebra structure on $\mathcal{G}^{*}$, such that $\left(\mathcal{G}, \mathcal{G}^{*}\right)$ form a Lie bialgebra. Conversely if $G$ is connected and simply connected, then every structure of Lie bialgebra $\left(\mathcal{G}, \mathcal{G}^{*}\right)$ defines a unique Poisson-Lie structure on $G$.

On the vector space $\mathcal{D}=\mathcal{G} \oplus \mathcal{G}^{*}$, there is a natural Lie algebra structure such that $\mathcal{G}$ and $\mathcal{G}^{*}$ are Lie subalgebras, whose bracket is

$$
[x, \alpha]=\operatorname{ad}_{x}^{*} \alpha-\operatorname{ad}_{\alpha}^{*} x
$$

where $x \in \mathcal{G}$ and $\alpha \in \mathcal{G}^{*}$. With that structure, $\mathcal{D}$ is called the double Lie algebra of $(G, \omega)$. 
For example, let $\mathcal{G}$ be a Lie algebra. Its dual space $\mathcal{G}^{*}$ is an Abelian Poisson-Lie group, where the Poisson bracket is

$$
\{\varphi, \psi\}(x)=\langle x,[\mathrm{~d} \varphi(x), \mathrm{d} \psi(x)]\rangle
$$

for all $\varphi, \psi \in C^{\infty}\left(\mathcal{G}^{*}\right)$. The Lie bialgebra of the Poisson-Lie group $\mathcal{G}^{*}$ is $\left(\mathcal{G}^{*}, \mathcal{G}\right)$, where the bracket of $\mathcal{G}^{*}$ is zero.

Proposition 8. Let $(G, \omega)$ be a Poisson-Lie group with Lie bialgebra $\left(\mathcal{G}, \mathcal{G}^{*}\right)$. Let $\mathcal{G} \dashv \mathcal{G}$ and $\mathcal{G}^{*} \vdash \mathcal{G}^{*}$ are the semi-direct products Lie algebras given above. Then $\left(\mathcal{G} \dashv \mathcal{G}, \mathcal{G}^{*} \vdash \mathcal{G}^{*}\right)$ has the structure of a Lie bialgebra.

Proof: By a simple calculation, we get

$$
\operatorname{ad}_{(x, y)}^{*}(\alpha, \beta)=\left(\operatorname{ad}_{x}^{*} \alpha+\operatorname{ad}_{y}^{*} \beta, \operatorname{ad}_{x}^{*} \beta\right)
$$

We need only to prove the relation of definition 7. Let $(x, y),\left(x^{\prime}, y^{\prime}\right) \in \mathcal{G} \times \mathcal{G}$ and $(\alpha, \beta) \in \mathcal{G}^{*} \times \mathcal{G}^{*}$. Since $\left(\mathcal{G}, \mathcal{G}^{*}\right)$ is a Lie bialgebra we have

$$
\begin{aligned}
&\left\langle\left[(x, y),\left(x^{\prime}, y^{\prime}\right)\right],\left[(\alpha, \beta),\left(\alpha^{\prime}, \beta^{\prime}\right)\right]\right\rangle \\
&=\left\langle\left(\left[x, x^{\prime}\right],\left[x, y^{\prime}\right]+\left[y, x^{\prime}\right]\right),\left(\left[\alpha, \beta^{\prime}\right]+\left[\beta, \alpha^{\prime}\right],\left[\beta, \beta^{\prime}\right]\right)\right\rangle \\
&=\left\langle\left[x, x^{\prime}\right],\left[\alpha, \beta^{\prime}\right]\right\rangle+\left\langle\left[x, x^{\prime}\right],\left[\beta, \alpha^{\prime}\right]\right\rangle+\left\langle\left[x, y^{\prime}\right],\left[\beta, \beta^{\prime}\right]\right\rangle+\left\langle\left[y, x^{\prime}\right],\left[\beta, \beta^{\prime}\right]\right\rangle \\
&=-\left[\operatorname{ad}_{x}^{*} \alpha, \beta^{\prime}\right]\left(x^{\prime}\right)-\left[\alpha, \operatorname{ad}_{x}^{*} \beta^{\prime}\right]\left(x^{\prime}\right)+\left[\operatorname{ad}_{x^{\prime}}^{*} \alpha, \beta^{\prime}\right](x)+\left[\alpha, \operatorname{ad}_{x^{\prime}}^{*} \beta^{\prime}\right](x) \\
&\left.-\left[\operatorname{ad}_{x}^{*} \beta, \alpha^{\prime}\right] x^{\prime}\right)-\left[\beta, \operatorname{ad}_{x}^{*} \alpha^{\prime}\right]\left(x^{\prime}\right)+\left[\operatorname{ad}_{x^{\prime}}^{*} \beta, \alpha^{\prime}\right](x)+\left[\beta, \operatorname{ad}_{x^{\prime}}^{*} \alpha^{\prime}\right](x) \\
&-\left[\operatorname{ad}_{x}^{*} \beta, \beta^{\prime}\right]\left(y^{\prime}\right)-\left[\beta, \operatorname{ad}_{x}^{*} \beta^{\prime}\right]\left(y^{\prime}\right)+\left[\operatorname{ad}_{y^{\prime}}^{*} \beta, \beta^{\prime}\right](x)+\left[\beta, \operatorname{ad}_{y^{\prime}}^{*} \beta^{\prime}\right](x) \\
&-\left[\operatorname{ad}_{y}^{*} \beta, \beta^{\prime}\right]\left(x^{\prime}\right)-\left[\beta, \operatorname{ad}_{y}^{*} \beta^{\prime}\right]\left(x^{\prime}\right)+\left[\operatorname{ad}_{x^{\prime}}^{*} \beta, \beta^{\prime}\right](y)+\left[\beta, \operatorname{ad}_{x^{\prime}}^{*} \beta^{\prime}\right](y) \\
&=-\left[\operatorname{ad}_{(x, y)}^{*}(\alpha, \beta),\left(\alpha^{\prime}, \beta^{\prime}\right)\right]\left(x^{\prime}, y^{\prime}\right)-\left[(\alpha, \beta), \operatorname{ad}_{(x, y)}^{*}\left(\alpha^{\prime}, \beta^{\prime}\right)\right]\left(x^{\prime}, y^{\prime}\right) \\
&+\left[\operatorname{ad}_{\left(x^{\prime}, y^{\prime}\right)}^{*}(\alpha, \beta),\left(\alpha^{\prime}, \beta^{\prime}\right)\right](x, y)+\left[(\alpha, \beta),\left[\operatorname{ad}_{(x, y)}^{*}(\alpha, \beta),\left(\alpha^{\prime}, \beta^{\prime}\right)\right](x, y) .\right.
\end{aligned}
$$

Then $\left(\mathcal{G} \dashv \mathcal{G}, \mathcal{G}^{*} \vdash \mathcal{G}^{*}\right)$ is a Lie bialgebra.

Definition 9. . Let $\left(G_{1}, \omega_{1}\right)$ and $\left(G_{2}, \omega_{2}\right)$, be two Poisson-Lie groups with Lie bialgebras $\left(\mathcal{G}_{1}, \mathcal{G}_{1}^{*}\right)$ and $\left(\mathcal{G}_{2}, \mathcal{G}_{2}^{*}\right)$. A Lie group morphism $\varphi: G_{1} \longrightarrow G_{2}$ is called a Poisson-Lie group morphism if it is also a Poisson map.

A Lie algebra morphism $f: \mathcal{G}_{1} \longrightarrow \mathcal{G}_{2}$ defines a Lie bialgebra morphism from $\left(\mathcal{G}_{1}, \mathcal{G}_{1}^{*}\right)$ to $\left(\mathcal{G}_{2}, \mathcal{G}_{2}^{*}\right)$, if its transposed map is also a Lie algebra morphism.

Let $\varphi:\left(G_{1}, \omega_{1}\right) \longrightarrow\left(G_{2}, \omega_{2}\right)$ be a Poisson-Lie group morphism. Then the tangent map $\varphi_{*}(e): \mathcal{G}_{1} \longrightarrow \mathcal{G}_{2}$ induces a Lie bialgebra morphism from $\left(\mathcal{G}_{1}, \mathcal{G}_{1}^{*}\right)$ to $\left(\mathcal{G}_{2}, \mathcal{G}_{2}^{*}\right)$. 
Proposition 10. Let $(G, \omega)$ be a Poisson-Lie group with Lie bialgebra $\left(\mathcal{G}, \mathcal{G}^{*}\right)$. Then $\left(\mathcal{G} \dashv \mathcal{G}, \mathcal{G}^{*} \vdash \mathcal{G}^{*}\right)$ is the Lie bialgebra of the Poisson-Lie group $\left(T G, \Omega_{T G}\right)$.

Proof: The projection

$$
\tau_{G}: T G \longrightarrow G: X_{g} \longmapsto g
$$

is a Poisson-Lie group morphism, where the Poisson structure of $G$ is zero. Then $\tau_{G}$ induces a Lie bialgebra morphism

$$
\tau_{G *}(e): \mathcal{G} \dashv \mathcal{G} \longrightarrow \mathcal{G}:(x, y) \longmapsto x
$$

where the bracket of $\mathcal{G}^{*}$ is also zero.

Hence, we have

$$
[(\alpha, 0),(\beta, 0)]=(0,0)
$$

for all $\alpha, \beta \in \mathcal{G}^{*}$.

Let

$$
\iota: \mathcal{G} \longrightarrow T G: x \longmapsto(e, x)
$$

where $(e, x) \in T_{e} G$ is regarded as element of $\mathcal{G}$. It is clear that $\iota$ is a Poisson-Lie group morphism from $\mathcal{G}$ to $T G$, where $\mathcal{G}$ is the linear Poisson-Lie group associated to the Lie algebra $\mathcal{G}^{*}$. Then

$$
\iota_{*}(0): \mathcal{G} \longrightarrow \mathcal{G} \times \mathcal{G}: x \longmapsto(0, x)
$$

induces a Lie bialgebra morphism from $\left(\mathcal{G}, \mathcal{G}^{*}\right)$ to $\left(\mathcal{G} \dashv \mathcal{G}, \mathcal{G}^{*} \vdash \mathcal{G}^{*}\right)$.

Then, for all $\alpha, \beta \in \mathcal{G}^{*}$, we have

$$
[(0, \alpha),(0, \beta)]=(0,[\alpha, \beta])
$$

For the last bracket $[(\alpha, 0),(0, \beta)]$, we need the following lemma.

Lemma 11. [12]. Let $(G, \omega)$ be a Poisson-Lie group and $\left(x_{i}\right)$ are local coordinates of $G$ in a neighborhood of e. For all $\alpha, \beta \in \mathcal{G}^{*}$ and $x \in \mathcal{G}$ we have

$$
[\alpha, \beta]_{\omega}(x)=\sum \frac{\partial \omega_{i j}}{\partial x_{k}}(e) \alpha_{i} \beta_{j} x_{k}
$$

where $\alpha=\Sigma \alpha_{i} \mathrm{~d} x_{i}$ and $\beta=\Sigma \beta_{i} \mathrm{~d} x_{i}$. 
We turn to the proof of the lemma. Let $\left(x_{i}\right)$ are local coordinates of $G$ in a neighborhood of $e$ and $\left(x_{i}, y_{j}\right)=\left(x_{i}, \dot{x}_{j}\right)$, the correspondent local coordinates of $T G$, in a neighberhood of $(e, o)$. By Remark 3, the Poisson bivector of $T G$ is expressed by

$$
\Omega(g, x)=\sum_{i j} \omega_{i j}(g) \frac{\partial}{\partial x_{i}} \wedge \frac{\partial}{\partial y_{j}}+\dot{\omega}_{i j}(x) \frac{\partial}{\partial y_{i}} \wedge \frac{\partial}{\partial y_{j}}
$$

Let $\alpha=\left(\alpha_{i}\right)$ and $\beta=\left(\beta_{j}\right)$ be elements of $\mathcal{G}^{*}$. We write

$$
(\alpha, 0)=\sum_{i} \alpha_{i} \mathrm{~d} x_{i} \quad \text { and } \quad(0, \beta)=\sum_{j} \beta_{j} \mathrm{~d} y_{j} .
$$

It follows from the lemma that

$$
[(\alpha, 0),(0, \beta)](x, y)=\sum_{i, j, k} \frac{\partial \omega_{i j}(e)}{\partial x_{k}} \alpha_{i} \beta_{j} x_{k}=[\alpha, \beta](x)
$$

for all $(x, y) \in \mathcal{G} \times \mathcal{G}$.

Hence

$$
[(\alpha, 0),(0, \beta)]=([\alpha, \beta], 0) .
$$

This concludes the proof of the proposition.

Corollary 12. Let $(G, \omega)$ be a Poisson-Lie group with dual group $G^{*}$. Then $T G^{*}$ is the dual group of the Poisson-Lie group $\left(T G, \Omega_{T G}\right)$, i.e., $(T G)^{*}=T\left(G^{*}\right)$.

Proof: It is clear that the map

$$
\rho: \mathcal{G}^{*} \times \mathcal{G}^{*} \longrightarrow \mathcal{G}^{*} \times \mathcal{G}^{*}, \quad \rho(\alpha, \beta) \longmapsto(\beta, \alpha)
$$

is a Lie bialgebra isomorphism from $\left(\mathcal{G}^{*} \dashv \mathcal{G}^{*}, \mathcal{G} \vdash \mathcal{G}\right)$ to $\left(\mathcal{G}^{*} \vdash \mathcal{G}^{*}, \mathcal{G} \dashv \mathcal{G}\right)$. According to Proposition $10,\left(\mathcal{G}^{*} \dashv \mathcal{G}^{*}, \mathcal{G} \vdash \mathcal{G}\right)$ is the Lie bialgebra of $T G^{*}$. Since $T G^{*}$ is connected and simply connected, $\rho$ can be integrated to an isomorphism of Poisson Lie groups from $T G^{*}$ to the dual of $\left(T G, \Omega_{T G}\right)$.

Proposition 13. Let $\mathcal{D}=\mathcal{G} \oplus \mathcal{G}^{*}$ be the double Lie algebra associated to $(G, \omega)$ and $\tilde{\mathcal{D}}=(\mathcal{G} \dashv \mathcal{G}) \oplus\left(\mathcal{G}^{*} \vdash \mathcal{G}^{*}\right)$ be the double Lie algebra associated to the Poisson-Lie group $\left(T G, \Omega_{T G}\right)$. Then $\tilde{\mathcal{D}}$ is isomorphic to the semi-direct product Lie algebra $\mathcal{D} \dashv \mathcal{D}$. 
Proof: We consider the map

$$
\begin{gathered}
f:(\mathcal{G} \dashv \mathcal{G}) \oplus\left(\mathcal{G}^{*} \vdash \mathcal{G}^{*}\right) \longrightarrow\left(\mathcal{G} \oplus \mathcal{G}^{*}\right) \times\left(\mathcal{G} \oplus \mathcal{G}^{*}\right) \\
(x, y)+(\alpha, \beta) \longmapsto(x+\beta, y+\alpha) .
\end{gathered}
$$

It suffices to show that $f$ is an isomorphism of Lie algebras from $\tilde{\mathcal{D}}$ to $\mathcal{D} \dashv \mathcal{D}$.

In fact, we have

$$
\begin{aligned}
f\left([(x, y),(\alpha, \beta)]_{\check{\mathcal{D}}}\right) & =f\left(\operatorname{ad}_{(x, y)}^{*}(\alpha, \beta)-\operatorname{ad}_{(\alpha, \beta)}^{*}(x, y)\right) \\
& =f\left(\left(-\operatorname{ad}_{\beta}^{*} x,-\operatorname{ad}_{\alpha}^{*} x-\operatorname{ad}_{\beta}^{*}(y)\right)+\left(\operatorname{ad}_{x}^{*} \alpha+\operatorname{ad}_{y}^{*} \beta, \operatorname{ad}_{x}^{*} \beta\right)\right) \\
& =\left(\operatorname{ad}_{x}^{*} \beta-\operatorname{ad}_{\beta}^{*} x, \operatorname{ad}_{x}^{*} \alpha-\operatorname{ad}_{\alpha}^{*} x+\operatorname{ad}_{y}^{*} \beta-\operatorname{ad}_{\beta}^{*} y\right) \\
& =([x, \beta],[x, \alpha]+[y, \beta])=[(x, y),(\beta, \alpha)]_{\mathcal{D} \dashv \mathcal{D}} \\
& =[f(x, y), f(\alpha, \beta)]_{\mathcal{D} \dashv \mathcal{D}}
\end{aligned}
$$

for all $x$ and $y \in \mathcal{G}, \alpha$ and $\beta \in \mathcal{G}^{*}$.

Similarly, we get

$$
\begin{aligned}
f\left(\left[(x, y),\left(x^{\prime}, y^{\prime}\right)\right]_{\tilde{\mathcal{D}}}\right) & =\left[f(x, y), f\left(x^{\prime}, y^{\prime}\right)\right]_{\mathcal{D} \dashv \mathcal{D}}, \\
f\left(\left[(\alpha, \beta),\left(\alpha^{\prime}, \beta^{\prime}\right)\right]_{\tilde{\mathcal{D}}}\right) & =\left[f(\alpha, \beta), f\left(\alpha^{\prime}, \beta^{\prime}\right)\right]_{\mathcal{D} \dashv \mathcal{D}} .
\end{aligned}
$$

Proposition 14. Let $H$ be a Poisson-Lie subgroup of $(G, \omega)$. Then $T H$ is also a Poisson-Lie subgroup of TG.

Proof: By definition, a Poisson-Lie subgroup of $G$ is a Lie subgroup $H$ of $G$, such that the injection map $\iota: H \longrightarrow G$, is a Poisson morphism.

It is clear that the tangent map $T \iota$ is a Lie group morphism from $T H$ to $T G$. Furthemore, by theorem 2, the injection map $T \iota$ is also a Poisson map. Hence $T H$ is a Poisson-Lie subgroup of $T G$.

\section{The Exact Case}

Now, we shall discuss an important example of Poisson Lie groups, which is the exact case. Throughout this section, we suppose that $G$ is connected.

A Poisson-Lie group $(G, \omega)$ is said to be exact, if the cocycle $\mathrm{d}_{e} \omega$ is a coboundary; i.e: there exists $r \in \mathcal{G} \wedge \mathcal{G}$ such that $\mathrm{d}_{e} \omega(x)=\operatorname{ad}_{x}(r)$, for all $x \in \mathcal{G}$. 
Let $r \in \mathcal{G} \wedge \mathcal{G}$, we define a bivector field on $G$ by

$$
\omega(g)=L_{g *} r-R_{g *} r, \quad \text { for all } g \in G .
$$

By Drinfel'd [1] [2], $(G, \omega)$ is a Poisson-Lie group if and only if the algebraic Schouten bracket [r,r] is invariant under the adjoint action of $G$ on $\mathcal{G} \wedge \mathcal{G} \wedge \mathcal{G}$.

Proposition 15. Let $(G, \omega)$ be an exact Poisson-Lie group with coboundary $\mathrm{d}_{e} \omega(x)=\operatorname{ad}_{x}(r)$, where

$$
r=\sum_{i j} r_{i j} r_{i} \wedge r_{j} \in \mathcal{G} \wedge \mathcal{G} .
$$

Then $\left(T G, \Omega_{T G}\right)$ is also an exact Poisson-Lie group with coboundary

$$
\mathrm{d}_{(e, 0)} \Omega(x, y)=\operatorname{ad}_{(x, y)}(\check{r})
$$

where

$$
\check{r}=\sum_{i j} r_{i j}\left(\left(r_{i}, 0\right) \wedge\left(0, r_{j}\right)+\left(0, r_{i}\right) \wedge\left(r_{j}, 0\right) \in \mathcal{G} \times \mathcal{G} \wedge \mathcal{G} \times \mathcal{G} .\right.
$$

Proof: We set $\varepsilon(x)=\mathrm{d}_{e} \omega(x)$, so that

$$
\varepsilon(x)(\alpha, \beta)=[\alpha, \beta](x)=\operatorname{ad}_{x}(r)(\alpha, \beta)=r\left(\operatorname{ad}_{x}^{*} \alpha, \beta\right)+r\left(\alpha, \operatorname{ad}_{x}^{*} \beta\right) .
$$

We also set $\check{\varepsilon}(x, y)=\mathrm{d}_{(e, 0)} \Omega(x, y)$.

Let $r=r_{1} \wedge r_{2}$, for all $\alpha, \beta, \alpha^{\prime}, \beta^{\prime} \in \mathcal{G}^{*}$ and $x, y \in \mathcal{G}$, we have

$$
\begin{aligned}
& \check{\varepsilon}(x, y)\left((\alpha, \beta),\left(\alpha^{\prime}, \beta^{\prime}\right)\right)=\left[(\alpha, \beta),\left(\alpha^{\prime}, \beta^{\prime}\right)\right](x, y) \\
= & {[\alpha, \beta](x)+\left[\beta, \alpha^{\prime}\right](x)+\left[\beta, \beta^{\prime}\right](y)=r_{1} \wedge r_{2}\left(\left(\operatorname{ad}_{x}^{*} \alpha, \beta^{\prime}\right)+\left(\alpha, \operatorname{ad}_{x}^{*} \beta^{\prime}\right)\right) } \\
& +r_{1} \wedge r_{2}\left(\left(\operatorname{ad}_{x}^{*} \beta, \alpha^{\prime}\right)+\left(\beta,\left(\operatorname{ad}_{x}^{*} \alpha^{\prime}\right)\right)+r_{1} \wedge r_{2}\left(\left(\left(\operatorname{ad}_{y}^{*} \beta, \beta^{\prime}\right)+\left(\beta,\left(\operatorname{ad}_{y}^{*} \beta^{\prime}\right)\right)\right.\right.\right. \\
= & r_{1}\left(\operatorname{ad}_{x}^{*} \alpha\right) r_{2}\left(\beta^{\prime}\right)-r_{2}\left(\operatorname{ad}_{x}^{*} \alpha\right) r_{1}\left(\beta^{\prime}\right)+r_{1}(\alpha) r_{2}\left(\operatorname{ad}_{x}^{*} \beta^{\prime}\right)-r_{2}(\alpha) r_{1}\left(\operatorname{ad}_{x}^{*} \beta^{\prime}\right) \\
& +r_{1}\left(\operatorname{ad}_{x}^{*} \beta\right) r_{2}\left(\alpha^{\prime}\right)-r_{2}\left(\operatorname{ad}_{x}^{*} \beta\right) r_{1}\left(\alpha^{\prime}\right)+r_{1}(\beta) r_{2}\left(\operatorname{ad}_{x}^{*} \alpha^{\prime}\right)-r_{2}(\beta) r_{1}\left(\operatorname{ad}_{x}^{*} \alpha^{\prime}\right) \\
& +r_{1}\left(\operatorname{ad}_{y}^{*} \beta\right) r_{2}\left(\beta^{\prime}\right)-r_{1}\left(\beta^{\prime}\right) r_{2}\left(\operatorname{ad}_{y}^{*} \beta\right)+r_{1}(\beta) r_{2}\left(\operatorname{ad}_{y}^{*} \beta^{\prime}\right) \\
& -r_{2}(\beta) r_{1}\left(\operatorname{ad}_{y}^{*} \beta^{\prime}\right)=r_{1}\left(\operatorname{ad}_{x}^{*} \alpha+\operatorname{ad}_{y}^{*} \beta\right) r_{2}\left(\beta^{\prime}\right)-r_{1}\left(\alpha^{\prime}\right) r_{2}\left(\operatorname{ad}_{x}^{*} \beta\right) \\
& +r_{1}(\alpha) r_{2}\left(\operatorname{ad}_{x}^{*} \beta^{\prime}\right)-r_{2}(\beta) r_{1}\left(\operatorname{ad}_{x}^{*} \alpha^{\prime}+\operatorname{ad}_{y}^{*} \beta^{\prime}\right)+r_{1}\left(\operatorname{ad}_{x}^{*} \beta\right) r_{2}\left(\alpha^{\prime}\right) \\
& -r_{1}\left(\beta^{\prime}\right) r_{2}\left(\operatorname{ad}_{x}^{*} \alpha+\operatorname{ad}_{y}^{*} \beta\right)+r_{2}\left(\operatorname{ad}_{x}^{*} \alpha^{\prime}+\operatorname{ad}_{y}^{*} \beta^{\prime}\right) r_{1}(\beta)-r_{2}(\alpha) r_{1}\left(\operatorname{ad}_{x}^{*} \beta^{\prime}\right) \\
= & \left(r_{1}, 0\right) \wedge\left(0, r_{2}\right)\left(\left(\operatorname{ad}_{x}^{*} \alpha+\operatorname{ad}_{y}^{*} \beta, \operatorname{ad}_{x}^{*} \beta\right),\left(\alpha^{\prime}, \beta^{\prime}\right)\right)
\end{aligned}
$$




$$
\begin{aligned}
& +\left(r_{1}, 0\right) \wedge\left(0, r_{2}\right)\left((\alpha, \beta),\left(\operatorname{ad}_{x}^{*} \alpha^{\prime}+\operatorname{ad}_{y}^{*} \beta^{\prime}, \operatorname{ad}_{x}^{*} \beta^{\prime}\right)\right) \\
& +\left(0, r_{1}\right) \wedge\left(r_{2}, 0\right)\left(\left(\operatorname{ad}_{x}^{*} \alpha+\operatorname{ad}_{y}^{*} \beta, \operatorname{ad}_{x}^{*} \beta\right),\left(\alpha^{\prime}, \beta^{\prime}\right)\right) \\
& +\left(0, r_{1}\right) \wedge\left(r_{2}, 0\right)\left((\alpha, \beta),\left(\operatorname{ad}_{x}^{*} \alpha^{\prime}+\operatorname{ad}_{y}^{*} \beta^{\prime}, \operatorname{ad}_{x}^{*} \beta^{\prime}\right)\right) \\
& =\left(\left(r_{1}, 0\right) \wedge\left(0, r_{2}\right)+\left(0, r_{1}\right) \wedge\left(r_{2}, 0\right)\right)\left(\left(\operatorname{ad}_{(x, y)}^{*}(\alpha, \beta),\left(\alpha^{\prime}, \beta^{\prime}\right)\right)\right. \\
& +\left((\alpha, \beta), \operatorname{ad}_{(x, y)}^{*}\left(\alpha^{\prime}, \beta^{\prime}\right)\right)=\operatorname{ad}_{(x, y)}(\check{r})\left((\alpha, \beta),\left(\alpha^{\prime}, \beta^{\prime}\right)\right)
\end{aligned}
$$

where

$$
\check{r}=\left(r_{1}, 0\right) \wedge\left(0, r_{2}\right)+\left(0, r_{1}\right) \wedge\left(r_{2}, 0\right) \in(\mathcal{G} \times \mathcal{G}) \wedge(\mathcal{G} \times \mathcal{G}) .
$$

For the general case: $r=\sum_{i j} r_{i j} r_{i} \wedge r_{j}$, we get

$$
\check{r}=\sum_{i j} r_{i j}\left(\left(r_{i}, 0\right) \wedge\left(0, r_{j}\right)+\left(0, r_{i}\right) \wedge\left(r_{j}, 0\right)\right) .
$$

Remark 16. If $G$ is connected and simply connected, the bivector $\omega$ is of the form

$$
\omega(g)=L_{g *} r-R_{g *} r
$$

where $[r, r]$ is $\mathrm{Ad}_{G}$-invariant. Since TG is also connected and simply connected, and as $\check{\varepsilon}$ is exact, the bivector $\Omega_{T G}$ is given by

$$
\Omega\left(X_{g}\right)=L_{X_{g} *} \check{r}-R_{X_{g} *} \check{r} .
$$

Furthermore $[\check{r}, \check{r}]$ is $\operatorname{Ad}_{T G}$-invariant.

\section{Poisson Action Lifting}

One of the fundamental notions related to Poisson-Lie groups is that of Poisson action. The famous example of dressing action [7], plays an important role for the description of the Poisson structure of $G$.

In this section, we will be interested in the lifting of Poisson actions.

Definition 17. A left action $\phi: G \times P \longrightarrow P$ of a Poisson-Lie group $(G, \omega)$ on a Poisson manifold $P$ is called a Poisson action, if it is a Poisson map with respect to the product Poisson structure on $G \times P$. 
Let $\phi: G \times P \longrightarrow P$ be a Poisson action of $G$ on $P$. Naturally, we have to regard the lifted action of $G$ on $T P$ given by

$$
\check{\phi}: G \times T P \longrightarrow T P:\left(g, u_{p}\right) \longmapsto \phi_{g *}\left(u_{p}\right)
$$

In the particular case, when $G$ is equipped with the trivial Poisson structure, $\phi$ is just an action of $G$ on $P$ by Poisson morphisms. Then $\check{\phi}$ is also an action of $G$ on $T P$ by Poisson morphisms. Since $G$ is trivial, $\check{\phi}$ is a Poisson action.

In the general case, this is not true. In fact, if $\phi$ is the left translation of $G$, for all $\varphi, \psi \in C^{\infty}(G), g, h \in G$ and $X_{h} \in T_{h} G$ we have

$$
\left\{\hat{\varphi}_{g}, \hat{\psi}_{g}\right\}\left(X_{h}\right)+\left\{\hat{\phi}_{X_{h}}, \hat{\psi}_{X_{h}}\right\}(g)=\left\{\left(\varphi \circ L_{g}\right),\left(\psi \circ L_{g}\right)\right\}\left(X_{h}\right)+\left\{\varphi \circ R_{h}, \psi \circ R_{h}\right\}(g) .
$$

Since $\{\hat{\varphi}, \hat{\psi}\}\left(L_{g *} X_{h}\right)=0, \check{\phi}$ is a Poisson action if and only if

$$
\left\{\varphi \circ R_{h}, \psi \circ R_{h}\right\}=0
$$

for all $\varphi, \psi \in C^{\infty}(G)$, i.e. $G$ is trivial.

For this reason, we will be interested in an other lifted action, that of TG on TP.

Theorem 18. Let $\phi: G \times P \longrightarrow P$ be a Poisson action of the Poisson-Lie group $(G, \omega)$ on a Poisson manifold $P$. We assume that $T P$ is equipped with the Poisson structure given in Theorem 2. Let

$$
\Phi: T G \times T P \longrightarrow T P:\left(X_{g}, u_{p}\right) \longmapsto T_{g, p} \phi\left(X_{g}, u_{p}\right)=\phi_{g *} u_{p}+\phi_{p *} X_{g}
$$

Then, $\Phi$ is a Poisson action of the Poisson-Lie group $\left(T G, \Omega_{T G}\right)$ on the Poisson manifold $\left(T P, \Omega_{T P}\right)$.

Proof: We know that the tangent map $T \phi: T(G \times P) \longrightarrow T P$ is a Poisson morphism. It suffices to show that the canonical bundle isomorphism

$$
\begin{gathered}
\rho: T(G \times P) \longrightarrow T G \times T P \\
X \longmapsto\left(\pi_{1 *} X, \pi_{2 *} X\right)
\end{gathered}
$$

is a Poisson morphism, where $\pi_{1}$ and $\pi_{2}$ are respectively the canonical projections from $G \times P$ on $G$ and $P$.

Let $\left(x_{i}\right)$ be local coordinates on $G$ and $\left(y_{j}\right)$ be local coordinates on P. Then $\rho$ sends the local coordinates $\left(x_{i}, y_{j}, \dot{x}_{i}, \dot{y}_{j}\right)$ of $T(G \times P)$ to the local coordinates $\left(\left(x_{i}, \dot{x}_{i}\right),\left(y_{j}, \dot{y}_{j}\right)\right)$ of $T G \times T P$. 
According to Remark 2 and Remark 3 and using the definition of direct Poisson structure, we have the following equalities:

$$
\begin{aligned}
& \left\{x_{i}, x_{j}\right\}_{T(G \times P)}=\left\{y_{i}, y_{j}\right\}_{T(G \times P)}=\left\{x_{i}, y_{j}\right\}_{T(G \times P)}=0 \\
& \left\{x_{i}, \dot{x}_{j}\right\}_{T(G \times P)}=\left\{x_{i}, x_{j}\right\}_{G \times P}=\left\{x_{i}, x_{j}\right\}_{G}=\omega_{i j}(x) \\
& \left\{y_{i}, \dot{y}_{j}\right\}_{T(G \times P)}=\left\{y_{i}, y_{j}\right\}_{G \times P}=\left\{y_{i}, y_{j}\right\}_{P}=t_{i j}(y) \\
& \left\{x_{i}, \dot{y}_{j}\right\}_{T(G \times P)}=\left\{x_{i}, \dot{y}_{j}\right\}_{G \times P}=0 \\
& \left\{\dot{x}_{i}, \dot{x}_{j}\right\}_{T(G \times P)}=\left\{x_{i}, x_{j}\right\}_{G \times P}=\dot{\omega}_{i j}(\dot{x}) \\
& \left\{\dot{y}_{i}, \dot{y}_{j}\right\}_{T(G \times P)}=\left\{y_{i}, y_{j}\right\}_{G \times P}=\dot{t}_{i j}(\dot{y}) \\
& \left\{\dot{x}_{i}, \dot{y}_{j}\right\}_{T(G \times P)}=\left\{x_{i}, y_{j}\right\}_{G \times P}=0 .
\end{aligned}
$$

Similarly, we get

$$
\begin{aligned}
& \left\{x_{i}, x_{j}\right\}_{T G \times T P}=\left\{y_{i}, y_{j}\right\}_{T G \times T P}=\left\{x_{i}, y_{j}\right\}_{T G \times T P}=0 \\
& \left\{x_{i}, \dot{x}_{j}\right\}_{T G \times T P}=\left\{x_{i}, \dot{x}_{j}\right\}_{T G}=\left\{x_{i}, x_{j}\right\}_{G}=\omega_{i j}(x) \\
& \left\{y_{i}, \dot{y}_{j}\right\}_{T G \times T P}=\left\{y_{i}, \dot{y}_{j}\right\}_{T P}=\left\{y_{i}, y_{j}\right\}_{P}=t_{i j}(y) \\
& \left\{x_{i}, \dot{y}_{j}\right\}_{T G \times T P}=0 \\
& \left\{\dot{x}_{i}, \dot{x}_{j}\right\}_{T G \times T P}=\left\{\dot{x}_{i}, \dot{x}_{j}\right\}_{T G}=\left\{x_{i}, x_{j} \dot{j}_{G}=\dot{\omega}_{i j}(\dot{x})\right. \\
& \left\{\dot{y}_{i}, \dot{y}_{j}\right\}_{T G \times T P}=\left\{\dot{y}_{i}, \dot{y}_{j}\right\}_{T P}=\left\{y_{i}, y_{j}\right\}_{P}=\dot{t}_{i j}(\dot{y}) \\
& \left\{\dot{x}_{i}, \dot{y}_{j}\right\}_{T G \times T P}=0 .
\end{aligned}
$$

The proof is completed.

Remark 19. If we consider the case of the left action of $G$ on itself we can deduce Theorem 5.

Example 20. Let

$$
\phi: G \times \mathcal{G}^{*} \longrightarrow \mathcal{G}^{*}:(g, \xi) \longmapsto \operatorname{Ad}_{g}^{*} \xi
$$

be the coadjoint action of $G$ on $g^{*}$. It is a Poisson action, when $G$ is equipped with the trivial Poisson structure and $\mathcal{G}^{*}$ with the linear Poisson structure.

We have

$$
\begin{gathered}
\Phi: T G \times T \mathcal{G}^{*} \longrightarrow T \mathcal{G}^{*} \\
\left(X_{g},(\xi, \eta)\right) \longmapsto\left(\phi_{g}(\xi), \phi_{g *}(\eta)+\phi_{\xi *} X_{g}\right) .
\end{gathered}
$$


Then

$$
\begin{gathered}
\Phi:(G \times \mathcal{G}) \times\left(\mathcal{G}^{*} \times \mathcal{G}^{*}\right) \longrightarrow \mathcal{G}^{*} \times \mathcal{G}^{*} \\
((g, x),(\xi, \eta)) \longmapsto\left(\operatorname{Ad}_{g}^{*} \xi, \operatorname{Ad}_{g}^{*} \eta+\phi_{\xi *}\left(R_{g *} x\right)\right) .
\end{gathered}
$$

On the other hand, we have:

$$
\begin{gathered}
\left(\phi_{\xi} \circ R_{g}\right)(h)=\operatorname{Ad}_{g h}^{*}(\xi)=\operatorname{Ad}_{h}^{*}\left(\operatorname{Ad}_{g}^{*} \xi\right) \\
\left(\phi_{\xi} \circ R_{g}\right)_{*}(x)=-\operatorname{ad}_{x}^{*}\left(\operatorname{Ad}_{g}^{*} \xi\right) .
\end{gathered}
$$

Consider the semi-direct product $G \dashv \mathcal{G}$. By duality and transposition, we obtain the following formula for the coadjoint action, which is valid for all $g \in G, x \in$ $\mathcal{G}, \xi \in \mathcal{G}$ and $\eta \in \mathcal{G}^{*}$

$$
\operatorname{Ad}_{(g, x)(\xi, \eta)}=\left(\operatorname{Ad}_{g}^{*} \xi-\operatorname{ad}_{x}^{*}\left(\operatorname{Ad}_{g}^{*}\right), \operatorname{Ad}_{g}^{*} \eta\right) .
$$

Corresponding to Example 6, $\mathrm{TG}^{*}$ is identified with $(\mathcal{G} \dashv \mathcal{G})^{*}$ by

$$
T \mathcal{G}^{*} \longrightarrow \mathcal{G}^{*} \times \mathcal{G}^{*}:(\xi, \eta) \longmapsto(\eta, \xi) .
$$

Since $G \dashv \mathcal{G}$ is also equipped with the null Poisson structure and since $\Phi$ is the coadjoint action associated to the semi product $G \dashv \mathcal{G}$, the map $\Phi$ is a Poisson action.

\section{Dressing Actions}

Example 20 is a particular case of dressing actions [5,7]. Let us recall this notion. In the following, we assume that $(G, \omega)$ is a simply connected Poisson-Lie group, with dual group $G^{*}$. Let $D$ be the simply connected Lie group, with Lie algebra $\mathcal{D}=\mathcal{G} \oplus \mathcal{G}^{*}$. By [7], the map

$$
\psi: G \times G^{*} \longrightarrow D:(g, u) \longmapsto g u
$$

is a local diffeomorphism. When it is a global diffeomorphism, $D$ is called a double Lie group. In this case, let $g \in G$ and $u \in G^{*}$, the product $u g$ can be uniquely written as : $u g=g^{u} u^{g}$, where $g^{u} \in G$ and $u^{g} \in G^{*}$. This define a left action of $G^{*}$ on $G$ by

$$
\phi: G^{*} \times G \longrightarrow G:(g, u) \longmapsto g^{u}
$$

and a right action of $G$ on $G^{*}$ by

$$
\phi^{\prime}: G^{*} \times G \longrightarrow G:(g, u) \longmapsto u^{g} .
$$

These actions are called dressing actions, they are Poisson actions. The orbits of $\phi$ and $\phi^{\prime}$ are respectively the symplectic leaves of $G$ and $G^{*}$. 


\section{Proposition 21.}

i) Assume that D is a double Lie group. Then TD is a double Lie group.

ii) Let

$$
\phi: G^{*} \times G \longrightarrow G:(g, u) \longmapsto g^{u}
$$

be the left dressing action of $G^{*}$ on $G$. Then the lifted action

$$
\Phi: T G^{*} \times T G \longrightarrow T G:\left(X_{u}, Y_{g}\right) \longrightarrow \phi_{u *} Y_{g}+\phi_{g *} X_{u}
$$

is exactly the left dressing action of $T G^{*}$ on $T G$.

\section{Proof:}

i) Since $D$ is a double Lie group, then

$$
T \psi: T G \times T G^{*} \longrightarrow T D:\left(X_{g}, Y_{u}\right) \longmapsto L_{g *} Y_{u}+R_{u *} X_{g}
$$

is a vector bundle isomorphism. Furthermore for all $X_{g}, Y_{u} \in T D$ we have

$$
X_{g} Y_{u}=L_{g *} Y_{u}+R_{u *} X_{g} .
$$

Hence $T D$ is a double Lie group associated to the Poisson-Lie group $T G$.

ii) By definition

$$
\Phi: T G^{*} \times T G \longrightarrow T G:\left(X_{u}, Y_{g}\right) \longrightarrow\left(g^{u}, \phi_{u *} Y_{g}+\phi_{g *} X_{u}\right) .
$$

On the other hand,

$$
u g=g^{u} u^{g}=\phi_{u}(g) . \phi_{u}^{\prime}(g)
$$

Then, we have

$$
L_{u *} Y_{g}=L_{g^{u} *} \phi_{u *}^{\prime} Y_{g}+R_{u^{g}} \phi_{u *} Y_{g} .
$$

Similarly, we have

$$
R_{g_{*}} X_{u}=L_{g^{u} *} \phi_{g *}^{\prime}\left(X_{u}\right)+R_{u^{g}} \phi_{g *}\left(X_{u}\right) .
$$

Hence

$$
\begin{aligned}
X_{u} Y_{g} & =L_{u *} Y_{g}+R_{g *} X_{u} \\
& =L_{g^{u} *}\left(\phi_{u *}^{\prime} Y_{g}+\phi_{g *}^{\prime} X_{u}\right)+R_{u^{g *}}\left(\phi_{u *} Y_{g}+\phi_{g *} X_{u}\right) \\
& =\left(g^{u}, \phi_{u *} Y_{g}+\phi_{g *} X_{u}\right)\left(u^{g}, \phi_{u *}^{\prime} Y_{g}+\phi_{g *}^{\prime} X_{u}\right) .
\end{aligned}
$$

Then the left dressing action of $T G^{*}$ on $T G$ is given by

$$
T G^{*} \times T G \longrightarrow T G:\left(X_{u}, Y_{g}\right) \longrightarrow\left(g^{u}, \phi_{u *} Y_{g}+\phi_{g *} X_{u}\right) .
$$

This conclued the proof. 


\section{Acknowledgements}

We would like to thank C.-M. Marle and M. Selmi, for their fruitful discussions and valuable suggestions.

\section{References}

[1] Drinfel'd.V., Hamiltonian Structures on Lie Groups, Lie Bialgebras and the Geometric Meaning of the Classical Yang-Baxter Equations, Soviet Math. Dokl. 27 (1983) 68-71.

[2] Drinfel'd V., Quantum Groups, Proc. ICM, Berkeley 1 (1986) 789-820.

[3] Drinfel'd V., On Poisson Homogeneous Space of Poisson-Lie Groups, Theor. Math. Phys. 95 (1993) 226-227.

[4] Kosmann-Schwarzbach Y. and Magri F., Poisson Lie Groups and Complete Integrability, Ann. Inst. Henri Poincaré Phys. Théor. 49 (1988) 433-460.

[5] Lu J-H., Multiplicative and Affine Poisson Structure on Lie Groups, Thesis, Berkeley, 1990.

[6] Lu J-H., Momentum Mappings and Reduction of Poisson Action. Symplectic Geometry, Groupoids and Integrable Systems, P. Dazord and A. Weinstein, Eds., Springer, 1991, pp. 209-226.

[7] Lu J-H. and Weinstein A., Poisson Lie Groups, Dressing Transformation and Bruhat Decomposition, J. Differential Geometry 31 (1990) 501-526.

[8] Lu J-H., Poisson Homogeneous Spaces and Lie Algebroids Associated to Poisson Action, Duke Math. J. 86 (1997) 261-304.

[9] Marsden J., Ratiu T. and Weinstein A., Semidirect Products and Reduction in Mechanics, Trans. Amer. Math. Soc. 28 (1984) 147-177.

[10] Sanchez de Alvarez G., Poisson Brackets and Dynamics, Dynamical Systems, Santiago, 1990, pp. 230-249.

[11] Semenov-Tian-Shansky M., Dressing Transformations and Poisson Lie Groups Actions, RIMS, Kyoto University 21 (1985) 1237-1260.

[12] Vaisman I., Lectures on the Geometry of Poisson Manifolds, Birkhäuser, Boston, 1994.

[13] Weinstein A., Some Remarks on Dressing Transformation, J. Fac. Sci. Univ. Tokyo Sect A Math. 36 (1988) 163-167.

Mohamed Boumaiza

Départemement de Mathématiques 
Faculté des sciences de Monastir 5019 Tunisia

E-mail address:

m_boumaiza2003@yahoo.fr

Nadhem Zaalani

Départemement de Mathématiques

Faculté des sciences de Monastir

5019 Tunisia

E-mail address:

n_zaalani@yahoo.fr 